\title{
Geometric Permutations and Common Transversals
}

\author{
M. Katchalski, ${ }^{1}$ T. Lewis, ${ }^{2}$ and A. Liu ${ }^{2}$ \\ ${ }^{1}$ Faculty of Mathematics, Technion, Haifa, Israel \\ ${ }^{2}$ Department of Mathematics, University of Alberta, Edmonton, Alberta, Canada T6G 2G1
}

\begin{abstract}
The object of this paper is to study how many essentially different common transversals a family of convex sets on the plane can have. In particular, we consider the case where the family consists of pairwise disjoint translates of a single convex set.
\end{abstract}

\section{Introduction}

We wish to count the number of different ways that straight lines can intersect a family of subsets of the plane. The following problem illustrates more clearly what is meant by "the number of different ways."

Let $C_{1}, C_{2}, \ldots, C_{n}$ be mutually disjoint convex subsets of the plane. Find the largest value of $p$ such that:

(i) there are $p$ straight lines which intersect all of the sets;

(ii) each of these $p$ lines intersects the sets in a different order.

If is any finite family of mutually disjoint convex subsets of the plane, any straight line which intersects each member of $\mathscr{F}$ meets them in a definite order, determining two permutations-one being the reverse of the other. The pair of permutations is called a geometric permutation of $\%$, and the family of all geometric permutations is denoted $h(\mathscr{F})$.

When consists of three congruent circles, $h(\mathscr{F})$ is either empty, or contains 1,2 , or 3 members as shown in Fig. 1. Since the maximum number of geometric permutations of $n$ sets is certainly no greater than $n ! / 2$, Fig. 1 completely solves the problem for three congruent circles.

It is interesting to note that if a fourth congruent circle is added to the problem, then the maximum number of geometric permutations would decrease. In other words, the maximum number of geometric permutations of $n$ sets does not depend only upon $n$. 

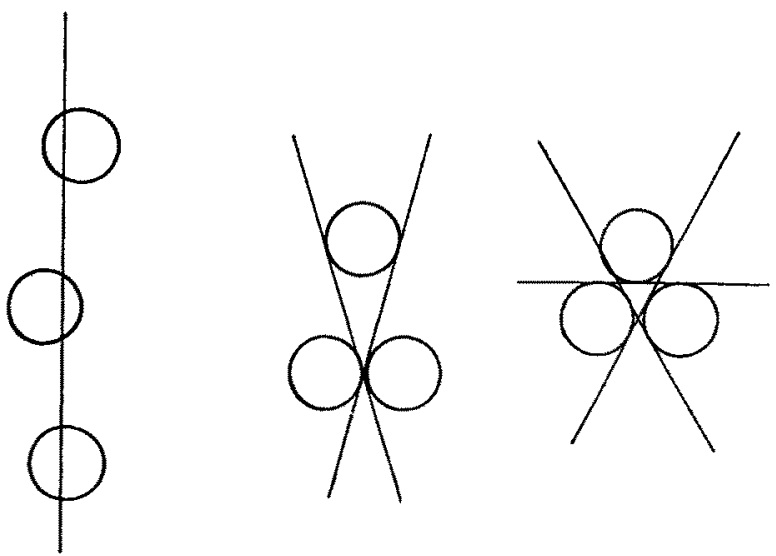

Fig. 1

In what follows, $F$ will always denote a nonempty family of mutually disjoint convex subsets of the plane. We will show that, even if the members of $\mathscr{F}$ are all congruent, without further restrictions on the size or shape of the members of $\mathscr{F}, \mu(\mathscr{F})$ can be made large by taking $\mathscr{F}$ to be sufficiently large. We will also show that the situation is radically different if all members of $\mathscr{F}$ are translates of the same convex set. In this case $\not(\mathscr{F})$ can be no greater than 8 provided $\mathscr{F}$ contains at least 11 members.

If $\mathscr{G}$ is any family of subsets of the plane (not necessarily convex or pairwise disjoint), then a straight line that meets each member of $\mathscr{G}$ is called a common transversal, or simply a transversal for $\mathscr{G}$. The question: "When does $\mathscr{G}$ have even one common transversal?" has not been fully solved (see [1] and [3] for a survey of some of the past results). In fact, it is this question that led us to consider the notion of geometric permutations, and one of us (Katchalski) has recently been able to apply the results of this paper to settle a question of Grünbaum [2] that was first raised almost 30 years ago. It should also be noted that the lower bound in Theorem 1 below has been improved to $2 n-2$ [4].

\section{Geometric Permutations of Arbitrary Families}

Theorem 1. For $n \geq 1$,

$$
n-1 \leq \max |\not(\mathscr{F})| \leq\left(\begin{array}{l}
n \\
2
\end{array}\right)
$$

where the maximum is taken over all families $\mathscr{F}$ of $n$ pairwise to disjoint convex sets in the plane.

To obtain the lower bound of Theorem 1, for each $n$ we will construct a family of $n$ congruent line segments admitting at least $n-1$ geometric permutations. (Figure 2 illustrates the case for $n=6$.) Place $n-1$ concurrent lines in the plane, labeling them $0,1, \ldots, n-2$ counterclockwise. Place line segment $S_{0}, \ldots, S_{n-2}$ so that they are mutually disjoint with $S_{i}$ extending from line $i$ to line $(i+n-2)$ $\bmod (n-1)$. Then place segment $S_{n-1}$ so that it extends from the point of 


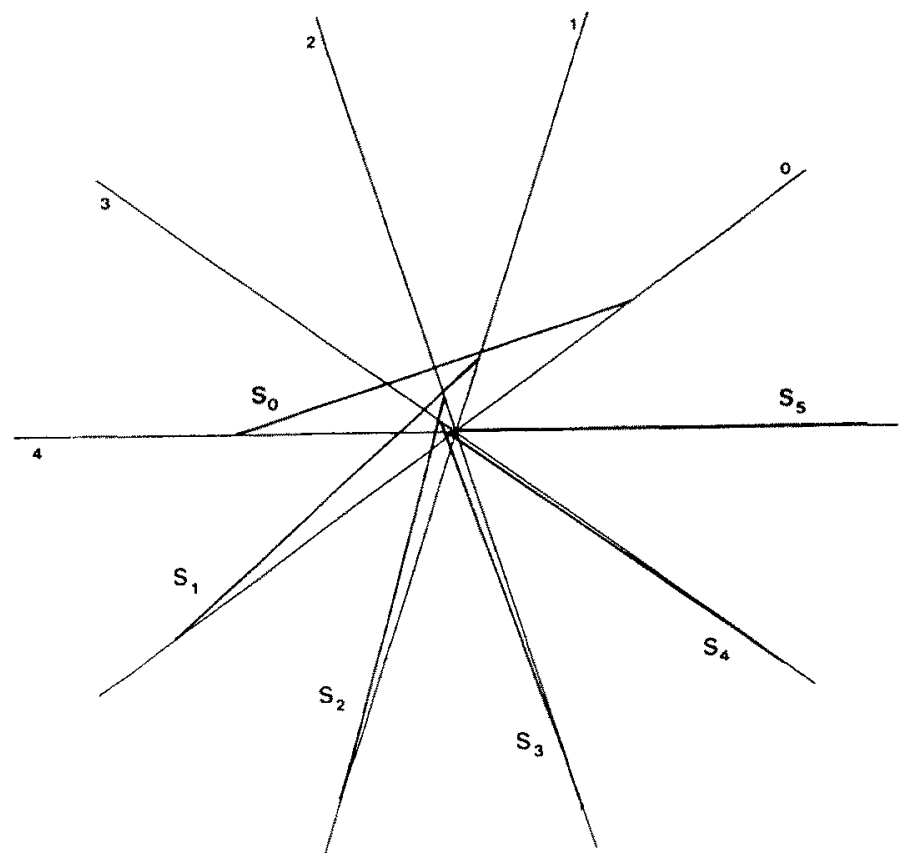

Fig. 2

concurrency along the line $n-2$ away from the other segments. Then the labeled lines are transversals for $\left\{S_{0}, S_{1}, \ldots, S_{n-1}\right\}$ which determine $n-1$ distinct geometric permutations. (A similar construction has been used in a different context in [7].)

We will use $T(F, G)$ to denote the collection of all transversals for the subsets $F$ and $G$, and $T(\mathscr{F})$ to denote the family of all transversals for $\mathscr{F}$.

If $t \in T(\mathscr{F})$, then $p_{t}$ is the geometric permutation determined by $t$. For a given geometric permutation, $p, T_{p}$ denotes the collection of all $t \in T(\mathscr{F})$ for which $p_{t}=p$.

To obtain the upper bound of Theorem 1 , it is convenient to use the notion of a symmetric twin, which is a symmetric subset of the unit circle consisting of either two disjoint arcs or a pair of antipodal points (see [4]). Symmetric twins arise in a natural way when considering common transversals: each transversal, $t$, for $F$ determines the symmetric twin, $\tilde{i}$, formed by intersecting the unit circle with the line through its center parallel to $t$. We shall use $\tilde{T}$ to denote the set $\{\tilde{t}: t \in T\}$, where $T$ is any family of transversals for $\mathscr{F}$.

Note that for $F, G \in \mathscr{F}, \tilde{T}(\{F, G\})$ is a symmetric twin since $F$ and $G$ are connected sets. The following lemma is implicitly contained in [6], and its proof, which is based on Helly's theorem on the line (see [5]), is omitted here.

Lemma 1. $\tilde{T}(\mathscr{F})=\bigcap\{\tilde{T}(\{F, G\}): F, G \in \mathscr{F}\}$.

Lemma 2. $|h(F)|$ is equal to the number of pairwise disjoint symmetric twins comprising $\tilde{T}(\mathscr{F})$. 
Proof. We first note that $\tilde{T}(\mathscr{F})=\bigcup\left\{\tilde{T}_{p}: p \in \not(\mathscr{F})\right\}$. We need to show that $\tilde{T}_{p}$ is a symmetric twin for every $p \in \not(\mathscr{F})$ and that for $p, q \in \not(\mathscr{F}), \tilde{T}_{p} \cap \tilde{T}_{q}=\varnothing$ unless $p=q$. The latter statement follows easily since two parallel common transversals of $\mathscr{F}$ determining distinct geometric permutations would contradict the assumption that members of $\mathscr{F}$ are pairwise disjoint.

Assume now that the first statement is false. Then there exists $\delta, t \in T_{p}$ such that $\tilde{j}$ and $\tilde{t}$ are separated by two symmetric twins $\tilde{u}$ and $\tilde{v}$ not in $\tilde{T}(\mathscr{F})$. Choose lines $\ell$ and $m$ such that $\tilde{\ell} \subset \tilde{u}$ and $\tilde{m} \subset \tilde{v}$. We may assume that $\ell$ and $m$ are the usual $x$-axis and $y$-axis, respectively.

Now $\ell$ separates some pair of sets $A, B \in \mathscr{F}$ and $m$ separates $C, D \in \mathscr{F}$. We may assume that $A$ is above $\ell$ and $B$ below, while $C$ is to the left of $m$ and $D$ to the right. In this setting, one of the common transversals, say $\sigma$, is ascending from left to right while the other, $t$, is descending from left to right. Now s will cut $B$ before $A$ and $C$ before $D$ while $t$ will also cut $C$ before $D$ but now $A$ before $B$. This contradicts the fact that $s$ and $t$ both determine the same permutation, $h$.

Lemmas 1 and 2 may be used to establish the upper bound of Theorem 1. By Lemma $1, \tilde{T}(\mathscr{F})$ is the intersection of $\left(\begin{array}{l}n \\ 2\end{array}\right)$ symmetric twins. Easy induction shows that the intersection of $k$ symmetric twins is a union of at most $k$ pairwise disjoint symmetric twins for any $k$. It follows that $\tilde{T}(\mathscr{F})$ is a union of at most $\left(\begin{array}{l}n \\ 2\end{array}\right)$ pairwise disjoint symmetric twins. Hence $|h(\mathscr{F})| \leq\left(\begin{array}{l}n \\ 2\end{array}\right)$ by Lemma 2 .

\section{Geometric Permutations of Translates}

Theorem 2. If $F$ is a family of at least 11 pairwise disjoint translates of a convex set, then $|h(\mathscr{F})| \leq 8$.

To prove Theorem 2, we require four lemmas about the possible arrangements of common transversals for families of translates.

Let $\ell$ and $m$ be two straight lines intersecting at a point $O$, dividing the plane into four quadrants. Let $Q$ be one of these quadrants and $S$ a convex set. We say that $S$ crosses $Q$ if $\ell \cap S \cap Q \neq \varnothing, m \cap S \cap Q \neq \varnothing$ and $O$ is not in $S$.

We first prove a preliminary result in the usual coordinate plane.

Lemma 3. Let $S$ be a convex set such that two translates $S_{1}$ and $S_{2}$ cross the first quadrant and two other translates $S_{3}$ and $S_{4}$ cross the second quadrant. Then either $S_{1} \cap S_{2} \neq \varnothing$ or $S_{3} \cap S_{4} \neq \varnothing$.

Proof. We may assume that the highest point where $S_{1} \cup S_{2} \cup S_{3} \cup S_{4}$ intersects the positive $y$-axis belongs to $S_{1}$. By translating it vertically upward if necessary, we may assume that $S_{1}$ is tangent to the positive $x$-axis. Let $O_{1}$ be a point of 
tangency, $A_{1}$ be the other point on the boundary of $S_{1}$ directly above $O_{1}$ and $B_{1}$ be a point of tangency to a supporting vertical line of $S_{1}$ on the right. We may assume that $O_{1} A_{1} B_{1}$ is not a degenerate triangle.

Let $O A B$ be the translate of $O_{1} A_{1} B_{1}$ with $O$ at the origin. Let $O_{3} A_{3} B_{3}$ be the triangle in $S_{3}$ and $O_{4} A_{4} B_{4}$ in $S_{4}$ corresponding to $O_{1} A_{1} B_{1}$ in $S_{1}$. Now $O_{3} A_{3} B_{3}$ must cross the second quadrant since $S_{3}$ does, and it does not intersect the positive $y$-axis above $A$. Similar conditions hold for $O_{4} A_{4} B_{4}$. Note that both $B_{3}$ and $B_{4}$ are in $O A B$.

Suppose the two triangles are disjoint. Then it would be possible to separate them by a line $\ell$, necessarily of positive slope. We may assume that $O_{3} A_{3} B_{3}$ is above $\ell$ and $O_{4} A_{4} B_{4}$ below. We now translate $O_{3} A_{3} B_{3}$ vertically upward until $B_{3}$ is on $A B$, and translate $O_{4} A_{4} B_{4}$ horizontally to the right until $B_{4}$ is on $O B$.

Since both triangles move away from $\ell$, they remain disjoint. Now $O O_{3}=B B_{3} \leq$ $A B=A_{4} B_{4}$, so that $O_{3} B_{3}$ must intersect $A_{4} B_{4}$, a contradiction. It follows that $S_{3}$ and $S_{4}$ are not disjoint.

Let two intersecting straight lines determine four quadrants and let $S$ be a convex set. Two opposite quadrants are called major quadrants if it is possible for two disjoint translates of $S$ to cross one of them. Two opposite quadrants are called minor quadrants if it is impossible for two disjoint translates of $S$ to cross either of them. Note that major and minor quadrants are defined with respect to $S$, but since no confusion will result, we suppress this reference.

Lemma 4. Let $\mathscr{F}$ be a family of at least six pairwise disjoint translates of a convex set with two nonparallel common transversals $\ell_{1}$ and $\ell_{2}$. Then two opposite quadrants they determine must be major quadrants and the other two minor quadrants. Moreover, if $\ell_{3}$ is another common transversal parallel to $\ell_{2}$, then the major quadrants determined by $\ell_{1}$ and $\ell_{3}$ are in corresponding positions to those determined by $\ell_{1}$ and $\ell_{2}$.

Proof. By using an affine transformation if necessary, we may assume that $\ell_{1}$ and $\ell_{2}$ are the usual coordinate axes. Now at most one translate can contain the origin. Hence at least two translates must cross some quadrant, say the first. By definition, the first and third quadrants are major quadrants. By Lemma 3, no two disjoint translates can cross the second quadrant. Using a reflection, along the line $y=x$, no two disjoint translates can cross the four quadrant either. Hence the second and fourth quadrants are minor quadrants. The second assertion of the lemma follows easily.

Let $\mathscr{F}$ be a family of pairwise disjoint translates of a convex set with two nonparallel common transversals $\ell$ and $m$. Now the symmetric twins $\not{\ell}$ and $m$ determine four arcs on the unit circle, giving rise to two nondegenerate symmetric twins. These correspond to the two pairs of opposite quadrants determined by $\ell$ and $m$. We shall denote by $\tilde{M}(\ell, m)$ the symmetric twin corresponding to the major quadrants. By Lemma $4, \tilde{M}(\ell, m)$ is well defined if $\mathscr{F}$ contains at least six members. 
Lemma 5. Suppose that $\ell_{1}, \ell_{2}, \ell_{3}$ are nonparallel transversals for $\mathscr{F}$, and suppose that $\mathscr{F}$ contains at least 11 members.

(i) If $\tilde{\ell}_{2} \subset \tilde{M}\left(\ell_{1}, \ell_{3}\right)$, then, $\tilde{M}\left(\ell_{1}, \ell_{3}\right)=\tilde{M}\left(\ell_{1}, \ell_{2}\right) \cup \tilde{M}\left(\ell_{2}, \ell_{3}\right)$.

(ii) In general $\tilde{M}\left(\ell_{1}, \ell_{3}\right) \subset \tilde{M}\left(\ell_{1}, \ell_{2}\right) \cup \tilde{M}\left(\ell_{2}, \ell_{3}\right)$.

Proof. (i) Translate $\ell_{2}$ to $\ell_{2}^{\prime}$ through the point of intersection $O$ of $\ell_{1}$ and $\ell_{3}$. Since $\tilde{\ell}_{2} \in \tilde{M}\left(\ell_{1}, \ell_{3}\right), \ell_{2}^{\prime}$ intersects all translates which cross the major quadrants determined by $\ell_{1}$ and $\ell_{3}$, as well as a translate which contains $O$. Since $\ell_{2}$ is a common transversal for $\mathscr{F}, \ell_{2}^{\prime}$ can miss at most one translate $F$. Now $\ell_{1}, \ell_{2}^{\prime}$ and $\ell_{3}$ are three concurrent common transversals for $\mathscr{F}-\{F\}$ which has at least ten members. It follows that we must have $\tilde{M}\left(\ell_{1}, \ell_{3}\right)=\tilde{M}\left(\ell_{1}, \ell_{2}^{\prime}\right) \cup \tilde{M}\left(\ell_{2}^{\prime}, \ell_{3}\right)$. By Lemma 4 , this is equivalent to statement (i).

(ii) This is true if, either $\tilde{\ell}_{2} \subset \tilde{M}\left(\ell_{1}, \ell_{3}\right)$ (by (i)), or if $\tilde{M}\left(\ell_{1}, \ell_{3}\right) \subset \tilde{M}\left(\ell_{1}, \ell_{2}\right)$ or $\tilde{M}\left(\ell_{1}, \ell_{3}\right) \subset \tilde{M}\left(\ell_{2}, \ell_{3}\right)$. If none of these cases occur, then all but at most three members cross the major quadrant determined by $\ell_{1}, \ell_{2}$, and all but at most three cross the major quadrant determined by $\ell_{2}, \ell_{3}$. Now consider the quadrants $Q$ determined by $\ell_{1}$ and $\ell_{3}$ that corresponds to $\tilde{M}\left(\ell_{1}, \ell_{2}\right) \cup \tilde{M}\left(\ell_{2}, \ell_{3}\right)$. There must be at least $|\mathscr{F}|-(3+3)+1$ members crossing this quadrant, for simple convexity considerations show that any member crossing the quadrants corresponding to $\tilde{M}\left(\ell_{1}, \ell_{2}\right)$ and $\tilde{M}\left(\ell_{2}, \ell_{3}\right)$ must either cross one of the quadrants $Q$ or else contain $\ell_{1} \cap \ell_{3}$.

Lemma 6. Let $\mathscr{F}$ be a family of at least 11 pairwise disjoint translates of a convex set with two nonparallel common transversals $\ell$ and $m$. If $\tilde{\ell}$ and $\tilde{m}$ are in the same component of $\tilde{T}(\mathscr{F})$, then $\tilde{M}(\ell, m) \subset \tilde{T}(\mathscr{F})$.

Proof. Using an affine transformation if necessary, we may assume that $\ell$ and $m$ are the usual coordinate axes and let the second and fourth quadrants be the major quadrants. Suppose $\tilde{M}(\ell, m) \not \subset \tilde{T}(\mathscr{F})$. Since $\ell$ and $m$ are in the same component of $\tilde{T}(\mathscr{F})$, for every positive number $\iota$, there is a common transversal for $F$ with slope 1 .

Let $t$ be such a common transversal. We claim that either $\tilde{M}(\ell, t)$ or $\tilde{M}(t, m)$ is contained in the first and third quadrants of the unit circle. If $\tilde{M}(\ell, t)$ is not, then $\tilde{m} \in \tilde{M}(\ell, t)$. By Lemma $5, \tilde{M}(\ell, t)=\tilde{M}(\ell, m) \cup \tilde{M}(t, m)$ and it follows that $\tilde{M}(t, m)$ is.

Let $t_{1}$ be a common transversal for $F$ with maximum positive slope $t_{1}$ such that $\tilde{M}\left(\ell, t_{1}\right)$ is contained in the first and third quadrants. Let $t_{2}$ be a common transversal for $F$ with minimum positive slope $t_{2}$ such that $\tilde{M}\left(t_{2}, m\right)$ is contained in the first and third quadrants.

If $t_{1}<t_{2}$, then there is a common transversal $t$ such that neither $\tilde{M}(\ell, t)$ nor $\tilde{M}(t, m)$ is contained in the first and third quadrants. Hence $t_{1} \geq t_{2}$. Now there is a common transversal $t$ such that $t \notin \tilde{M}(\ell, m)$ but $\tilde{M}(\ell, m) \not \subset \tilde{M}(\ell, t) \cup \tilde{M}(t, m)$. This contradicts Lemma 5 . 


\section{Proof of Theorem 2}

By Lemma $2,|h(\mathscr{F})|$ is given by the number of pairwise disjoint symmetric twins comprising $\tilde{T}(\mathscr{F})$. Let $k=|h(\mathscr{F})|$. For $1 \leq i \leq k$, let the $i$ th symmetric twin be bounded by $\tilde{\ell}_{2 i-1}$ and $\tilde{\ell}_{2 i}$. By Lemma $6, \tilde{T}(\mathscr{F})=\bigcup\left\{\tilde{M}\left(\ell_{2 i-1}, \ell_{2 i}\right): 1 \leq i \leq \ell\right\}$. Denote by $\tilde{L}\left(\ell_{2 i}, \ell_{2 i+1}\right)$ the symmetric twin bounded by $\tilde{\ell}_{2 i}$ and $\tilde{\ell}_{2 i+1}$ which is not contained in $\tilde{T}(\mathscr{F})$. Since each of $\tilde{L}\left(\ell_{2 i-1}, \ell_{2 i}\right)$ is a major quadrant, it cannot be that each of $\tilde{L}\left(\ell_{2 i}, \ell_{2 i+1}\right)$ is also a major quadrant, else by Lemma 5 , none of the lines $\ell_{i}, \ell_{j}$ would yield a minor quadrant. For definiteness, we will suppose that $L\left(\ell_{1}, \ell_{2 \ell}\right)$ corresponds to the minor quadrant determined by $\ell_{1}$ and $\ell_{2 \ell}$.

Now there are at most three translates which can fail to cross the major quadrants determined by $\ell_{1}$ and $\ell_{2 k}$, namely, $F_{1}$ crossing one minor quadrant, $F_{2}$ crossing the other and $F_{3}$ containing the point of intersection of $\ell_{1}$ and $\ell_{2 \ell}$. By Lemma 3, using an affine transformation if necessary, at most two other translates $G_{1}$ and $G_{2}$ can intersect $\ell_{1}$ between $\ell_{1} \cap F_{1}$ and $\ell_{1} \cap F_{2}$ or intersect $\ell_{2 A}$ between $\ell_{2 \ell} \cap F_{1}$ and $\ell_{2 \ell} \cap F_{2}$.

The only pairs $\{F, G\}, F, G \in \mathscr{F}$, such that $\tilde{T}(\{F, G\})$ do not contain $\tilde{M}\left(\ell_{1}, \ell_{2 \ell}\right)$ are $\left\{F_{1}, G_{1}\right\},\left\{F_{1}, G_{2}\right\},\left\{F_{2}, G_{1}\right\},\left\{F_{2}, G_{2}\right\},\left\{F_{1}, F_{2}\right\},\left\{F_{1}, F_{3}\right\}$ and $\left\{F_{2}, F_{3}\right\}$. Now $\tilde{T}(\mathscr{F})$ is the intersection of the above seven symmetric twins with $\tilde{M}\left(\ell_{1}, \ell_{2 \ell}\right)$. Hence $|h(\mathscr{F})| \leq 8$.

\section{Related Problems}

It may be of interest to sharpen the results of Theorems 1 and 2 . In Theorem 1, perhaps the gap between $n-1$ and $\left(\begin{array}{l}n \\ 2\end{array}\right)$ can be narrowed. In Theorem 2 , one may seek a characterization of all finite numbers $k$ for which $|h(\mathscr{F})|=\boldsymbol{k}$ for some family $\mathscr{F}$ of convex sets in the plane with $|\mathscr{F}|$ increasing without bound. Analogues of Theorems 1 and 2 in Euclidean spaces of higher dimensions may be developed.

Finally, we raise a combinatorial problem. Given a family $F$ of permutations of $\{1,2, \ldots, n\}$, what are the conditions which will guarantee the existence of a family $\mathscr{F}$ of $n$ pairwise disjoint translates of a convex set such that $h \subset n(\mathscr{F})$ ?

\section{References}

1. L. Danzer, B. Grünbaum, and V. Klec, Helly's theorem and its relatives, Proc. Sympos. Pure Math. 7 (1962), 101-180.

2. B. Grünbaum, On common transversals, Arch. Math. 9 (1958), 465-469.

3. H. Hadwiger, H. Debrunner, and V. Klee, Combinatorial Geometry in the Plane, Holt, Rinehart and Winston, New York, 1964.

4. M. Katchalski, T. Lewis, and J. Zaks, Geometric permutations for convex sets, Discrete Math, to appear.

5. M. Katchalski and A. Liu, A problem of geometry in $\boldsymbol{R}^{n}$, Proc. Amer. Math. Soc. 75 (1979), 284-288.

6. M. Katchalski and A. Liu, Symmetric twins and common transversals, Pacific J. Math. 86 (1980), 513-515.

7. T. Lewis, Two counterexamples concerning transversals for convex subsets of the plane, Geom. Dedicata 9 (1980), 461-465.

Received October 18, 1984, and in revised form May 1, 1985. 